\title{
Intranasal Delivery of Antiepileptic Medications for Treatment of Seizures
}

\author{
Daniel P. Wermeling \\ Department of Pharmacy Practice and Science, University of Kentucky College of Pharmacy, Lexington, Kentucky 40536-0082
}

\begin{abstract}
Summary: Acute isolated seizure, repetitive or recurrent seizures, and status epilepticus are all deemed medical emergencies. Mortality and worse neurologic outcome are directly associated with the duration of seizure activity. A number of recent reviews have described consensus statements regarding the pharmacologic treatment protocols for seizures when patients are in pre-hospital, institutional, and home-bound settings. Benzodiazepines, such as lorazepam, diazepam, midazolam, and clonazepam are considered to be medications of first choice. The rapidity by which a medication can be delivered to the systemic circulation and then to the brain plays a significant role in reducing the time needed to treat seizures and reduce opportunity for damage to the CNS. Speed of delivery, particularly outside of the hospital, is enhanced when transmucosal routes of delivery are used in place of an intravenous injection.

Intranasal transmucosal delivery of benzodiazepines is useful in reducing time to drug administration and cessation of seizures in the pre-hospital setting, when actively seizing pa-
\end{abstract}

tients arrive in the emergency room, and at home where caregivers treat their dependents. This review summarizes factors to consider when choosing a benzodiazepine for intranasal administration, including formulation and device considerations, pharmacology and pharmacokinetic/pharmacodynamic profiles. A review of the most relevant clinical studies in epilepsy patients will provide context for the relative success of this technique with a number of benzodiazepines and relatively less sophisticated nasal preparations. Neuropeptides delivered intranasally, crossing the blood-brain barrier via the olfactory system, may increase the availability of medications for treatment of epilepsy. Consequently, there remains a significant unmet medical need to serve the pharamcotherapeutic requirements of epilepsy patients through commercial development and marketing of intranasal antiepileptic products. Key Words: Intranasal, drug delivery, antiepileptic medications, treatment of seizures, emergency pharmacotherapy, benzodiazepines, blood-brain barrier.

\section{INTRODUCTION}

Patients with a life-threatening or unstable condition presenting to pre-hospital, emergency service field personnel or hospital emergency facilities will have an intravenous (IV) line established for rapid administration of medications. Organized health care has developed standards of care and assessment and treatment protocols for a variety of conditions to stabilize the patient as rapidly as possible. Clearly, patients experiencing epileptic seizures have a medical emergency and require prompt medical care. ${ }^{1-4}$ Several consensus statements have been published describing pharmacologic treatment protocols for patients presenting with seizures or in status epilepticus. The guidelines provide recognition of the need for prompt treatment and recommendations for var-

Address correspondence and reprint requests to: Daniel P. Wermeling, Pharm.D., Associate Professor, Department of Pharmacy Practice and Science, University of Kentucky College of Pharmacy, Lexington, KY 40536-0082. E-mail: dwermel@uky.edu. ious transmucosal routes of benzodiazepine delivery when IV access has not yet been established. ${ }^{5-9}$ The marketing of diazepam rectal gel is made in recognition of the need for noninjection-based delivery; however, the aesthetics of rectal delivery are not popular with patients and caregivers.

Intranasal administration of antiepileptic medications, in particular benzodiazepines, has been studied with various preparations. Intranasal midazolam has been extensively studied in epilepsy patients and is recommended in some consensus guidelines as an alternative drug delivery technique for prompt treatment. ${ }^{10-12}$

This review provides an overview of the considerations necessary for nasal delivery of antiepileptic medications. An understanding of nasal anatomy and physiology is required to appreciate the operating limitations of intranasal drug administration. Ad-hoc preparations or products for registration must consider designs that integrate the medication and its chemistry, the formulation, and the administration device to work successfully. Early 
stage studies in healthy volunteers are important steps in determining if appropriate pharmacokinetic and pharmacodynamic properties and safety profile will lend themselves to treating seizures. Clinical experience in treating epileptic patients with various preparations suggests this is a rapid-acting and effective drug-delivery alternative.

\section{Nasal anatomy as related to intranasal drug delivery}

Intranasal sprays of medication intended for systemic drug absorption are generally designed to target the turbinates on the medial wall of the nasal cavity (FIG. 1). The turbinates serve as a baffle in which inspired air is humidified and filtered. This region of the nasal cavity is covered with a thin mucus layer, a monolayer ciliated epithelium, with an abundant underlying blood supply. ${ }^{13}$ These conditions are ideal to permit passive diffusion (transcellular) of medications with certain chemical characteristics across cell membranes and into the bloodstream. Some medications also transit to the bloodstream by passing through the tight-cell junctions between cells (paracellular).

To reach the turbinates, the nasal spray device must be inserted fully into the nasal vestibule with the atomizer tip placed at the nasal valve, and then aimed laterally toward the turbinates. ${ }^{14}$ Activation of the device ejects

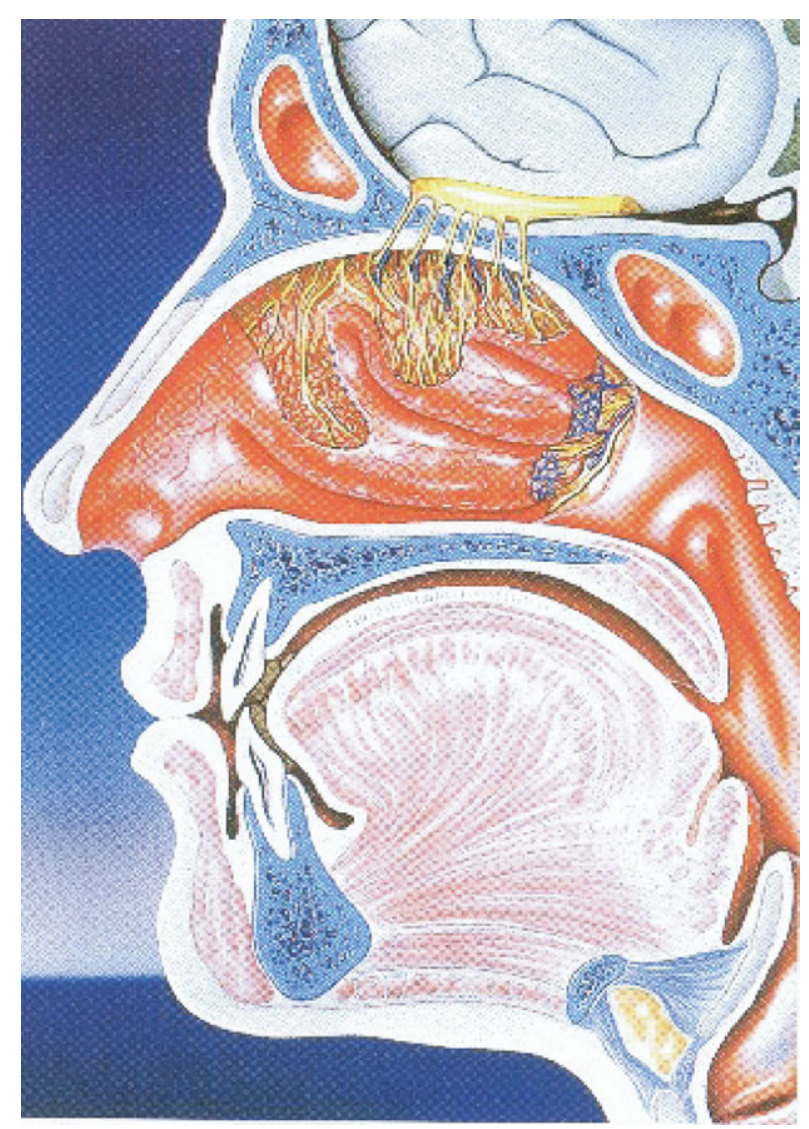

FIG. 1. Anatomy of the nasal cavity and relation to the brain. the liquid as an atomized spray or plume. The bulk of the spray impacts the anterior and inferior portions of the nasal cavity as a simple ballistic missile. The smallest particles, less than 10 microns in size, may be carried by air currents more superiorly in the nasal cavity and impact on the superior turbinate and possibly reach the olfactory region and nerve. ${ }^{15,16}$ There is substantial evidence in animals, and some evidence in man, that the olfactory nerve can absorb or actively transport medications to the central nervous system via the olfactory bulb (nose to brain theory). Differences in animal and human nasal apparatus anatomy, and certain characteristics of the medication, seem to play roles as to whether medication is transported to the brain via this mechanism, and if a pharmacologic effect is observed. ${ }^{17}$

Under ideal conditions, most medication is absorbed from the nasal cavity and into the bloodstream within 15 to 20 minutes, thus generally avoiding the first-pass gut metabolism. ${ }^{18}$ Medication remaining in the nasal cavity beyond this time is subject to elimination via various enzyme systems present within the nasal mucus and by swallowing. A second absorption phase can be observed with nasally administered medications having incomplete nasal absorption that are not subject to high firstpass gut metabolism.

Nasal physiologic changes during pathologic conditions, such as polyposis and allergic and vasomotor rhinitis, could theoretically alter the biopharmaceutics of intranasal medications intended for systemic drug administration. ${ }^{13}$ Physical obstruction of the nasal passage(s) due to prior trauma and subsequent deflection of the passageways is another possibility. Last, increases in mucus production and changes in mucociliary clearance rates could affect bioavailability.

Pharmaceutical regulatory agencies have required studies of the effect of rhinitis on nasal drug delivery biopharmaceutics. It has been demonstrated that there is a lack of effect of nasal mucosal inflammation on the absorption of hydromorphone, butorphanol, buserelin, and triamcinolone acetonide, with the exception reported for desmopressin. ${ }^{13,19,20}$ Inconsistent results have been reported on the biopharmaceutical disposition of these medications when pretreatment with oral or topical decongestants was administered. Small but statistically measurable changes in rate or extent of absorption have been reported when decongestants were coadministered. $^{13}$

Increased nasal mucus production is commonly observed with actively seizing patients and could be clinically relevant to intranasal drug administration for treatment of seizures. Holsti et al. ${ }^{21}$ recognized this consideration in their pre-hospital treatment protocol that called for suction of mucus from the nasal cavity prior to administration of intranasal midazolam. Pre-treatment nasal cavity suctioning was likely contributory to the success observed in rapidly 
aborting seizure activity after intranasal midazolam administration.

\section{DESIGN OF NASAL PRODUCTS FOR SYSTEMIC DRUG ADMINISTRATION}

Many intranasal delivery products are designed to serve certain purposes or unmet medical needs. Clearly, a nasal spray can remove the needle from drug administration, as is the case with the conversion of the protein calcitonin from a daily injection to a nasal spray. Furthermore, beyond just removing the needle from delivery, intranasal products are designed for rapid action, such as those products designed to treat migraine headache (sumatriptan, butorphanol, zolmitriptan, and dihydroergotamine) or pain in general (fentanyl, hydromorphone). ${ }^{18,22}$

What makes each of the aforementioned products successful is that their design satisfied several fundamentals necessary for intranasal delivery (Table 1 ) ${ }^{18}$ First, there must be a selection of drug candidates based on their pharmacologic and therapeutic properties. In the case of benzodiazepines, there are at least four reasonable alternatives to consider: 1) diazepam, 2) lorazepam, 3) clonazepam, and 4) midazolam. The rationale for choosing one versus another based purely on pharmacology is beyond the scope of this article. However, ease of passing the blood-brain barrier, receptor occupancy, speed of absorption, and clearance rate properties must be considered. One may also wish to consider choices based on prior clinical performance and current marketing within a Food and Drug Administration-approved label for treatment of acute seizures (e.g., diazepam and lorazepam are qualified in this regard).

Physical-chemical properties of the candidates must also be considered. Water-solubility is important for formulation considerations. Log $\mathrm{P}$, derived from the octanol/water partition coefficient, is a surrogate for lipophilicity, and a potential for compounds to diffuse across biologic membranes. Unfortunately, benzodiazepines have limited water solubility at $\mathrm{pH} 5$ to 7 , the $\mathrm{pH}$ of nasal secretions, and where most nasal solutions are formu-

Table 1. Chemistry and Formulation Issues Affecting Intranasal Medication Bioavailability and Tolerability

- Potent medication, $<20 \mathrm{mg}$ per dose

- Molecular weight, $<1000$ Daltons

- Excellent water solubility

- $\mathrm{pKa}$ and $\mathrm{pH}$ control of aqueous solutions

- Osmolality-isotonic to slightly hypertonic

- Stability in processing and storage

- Compatibility with sprayer components

- Use of special excipients to manage

- Solubility

Stability

- Permeation lated. These medications have sufficient lipophilic character to readily cross biologic membranes.

Benzodiazepines satisfy the potency requirement in that typical doses are all less than $20 \mathrm{mg}$. The dose must have sufficient solubility to be administered in approximately $100 \mu \mathrm{L}$ to $200 \mu \mathrm{L}$ (1 spray per naris) of solution. The nasal cavity can retain 100 to $150 \mu \mathrm{L}$ without causing immediate run-off out the front of the nose or down the nasopharynx. Water is the preferred solvent, but many medications, including the benzodiazepines, do not have sufficient water solubility to formulate a nasal product. Although midazolam injection is prepared in an aqueous solvent system, the solution is buffered to an irritating $\mathrm{pH} 3$ as a requirement to maintain drug in solution and to prevent instability of the core-ring structure from uncoupling. Additional solubilization strategies are necessary, including the use of organic co-solvents, excipients, such as cyclodextrins or other agents to from water-soluble inclusion complexes or preparation of emulsions.

Design of the formulation must account for other factors as well. It is useful to design the formulation to be isotonic to slightly hypertonic to optimize absorption and tolerability. Viscosity-enhancing agents, such as methylcellulose, can promote retention in the nasal cavity by slowing the ciliary movement of mucus. Surfactants or polymers can be used to enhance transmembrane permeation. Last, the drug and formulation have to be stable in the device during processing (i.e., sterilization and storage, thus possibly requiring stabilizers).

The choice of delivery device for the medication is another critical consideration. FIG. 2 depicts a number of different nasal spray devices. Squeeze bottles are available, but have no metering device appropriate to administer potent systemic medications such as benzodiazepines. Multi-dose bottles are available for chronic drug administration; this is not a likely consideration regarding seizure emergencies. A standard syringe with a Luer fitting to accept a nasal atomizer has been used to draw up and administer injection-based drug solutions into the nasal cavity for opiate overdoses, acute pain, and to deliver midazolam injection to the nasal cavity of a seizing patient. Unit-dose devices similar to those used for migraine treatment are also available and being used in development of benzodiazepine nasal spray products. The choice of device depends on factors such as intended clinical use, setting, and stability with the drug and formulation, among others.

Ideally, a well-designed formulation must not induce localized toxicity with acute or chronic use. For example, chronic vasoconstriction, irritation, or inflammation can induce tissue damage, including ulceration, epistaxis, nasal-septal defects, and fistulae. Formulations should not cause damage to the cilia. ${ }^{23}$ Due to the water insoluble nature of benzodiazepines, a common approach to nasal 




FIG. 2. Representative nasal sprayers.

delivery formulations is to use organic solvents or cosolvents with water. For acute use there is a risk of transient irritation from such formulations. Although not ideal, the risk-benefit assessment likely still favors use of such products in treatment of life-threatening circumstances such as seizures. Chronic or daily use of an irritating product could lead to more serious sequelae from nasal delivery.

The concept of direct entrance to the CNS has been considered for nasal drug delivery. ${ }^{15-17}$ Neuroproteins ${ }^{24}$ and other medications that do not readily cross the bloodbrain barrier after systemic administration are considered targets for this drug delivery application. The challenges with nose to brain transit of medications are that: 1) the medication must be very potent, 2) the device must optimize an ability of the spray to reach the most superior portions of the nasal cavity, 3 ) sufficient medication must reach the olfactory nerve, 4) a mechanism of transport must be available, and 5) the medication must reach relevant brain structures and be able to diffuse through the brain parenchyma to the relevant receptors.

\section{PHARMACOKINETICS AND PHARMACODYNAMICS OF NASAL BENZODIAZEPINES IN HEALTHY VOLUNTEERS}

The first studies of intranasal delivery of benzodiazepine formulations were performed in healthy volunteers. The purpose of the trials was to generate systemic exposure and local tolerance data for various formulation approaches. Table 2 provides some comparative data that

Table 2. Pharmacokinetics of Selected Benzodiazepine Formulations Administered Intranasally

\begin{tabular}{lclccc}
\hline \multicolumn{1}{c}{ Drug } & Dose $(\mathrm{mg})$ & \multicolumn{1}{c}{ Formulation } & $\mathrm{C}_{\max }(\mathrm{ng} / \mathrm{mL})$ & $\mathrm{t}_{\max }(\mathrm{min})$ & $\mathrm{F}(\%)$ \\
\hline Clonazepam & 1.0 & B-CDE/ $\mathrm{H}_{2} \mathrm{O}$ & 6.3 & 17.5 & 45 \\
Lorazepam & 2.0 & Prop. Gly. & 21.4 & 30 & 77.7 \\
Lorazepam & 4.0 & Cremophor El & 18.7 & 135 & 51 \\
Midazolam & 5.0 & PEG 400/Prop. Gly. & 80 & 10 & 72.5 \\
Midazolam & 5.0 & Prop. Gly./H $\mathrm{H}_{2} \mathrm{O}$ & 71 & 14 & 83 \\
Midazolam & 3.4 & SBE-CDE & 51 & 15 & 71 \\
Midazolam & $0.25 \mathrm{mg} / \mathrm{kg}$ & Aqueous injection & 147 & 25 & 50 \\
Diazepam & 7.0 & PEG 300 & 179 & 42 & 42 \\
\hline
\end{tabular}

$\mathrm{C}_{\max }=$ maximum plasma concentration; $\mathrm{F}=$ absolute bioavailability comparing nasal dose to an intravenous dose; $\mathrm{PEG}=$ polyethylene glycol; Prop. Gly. $=$ propylene glycol; $\mathrm{t}_{\max }=$ time to maximum plasma concentration; SBE-CDE $=$ sulfobutylether-beta-cyclodextrin. 


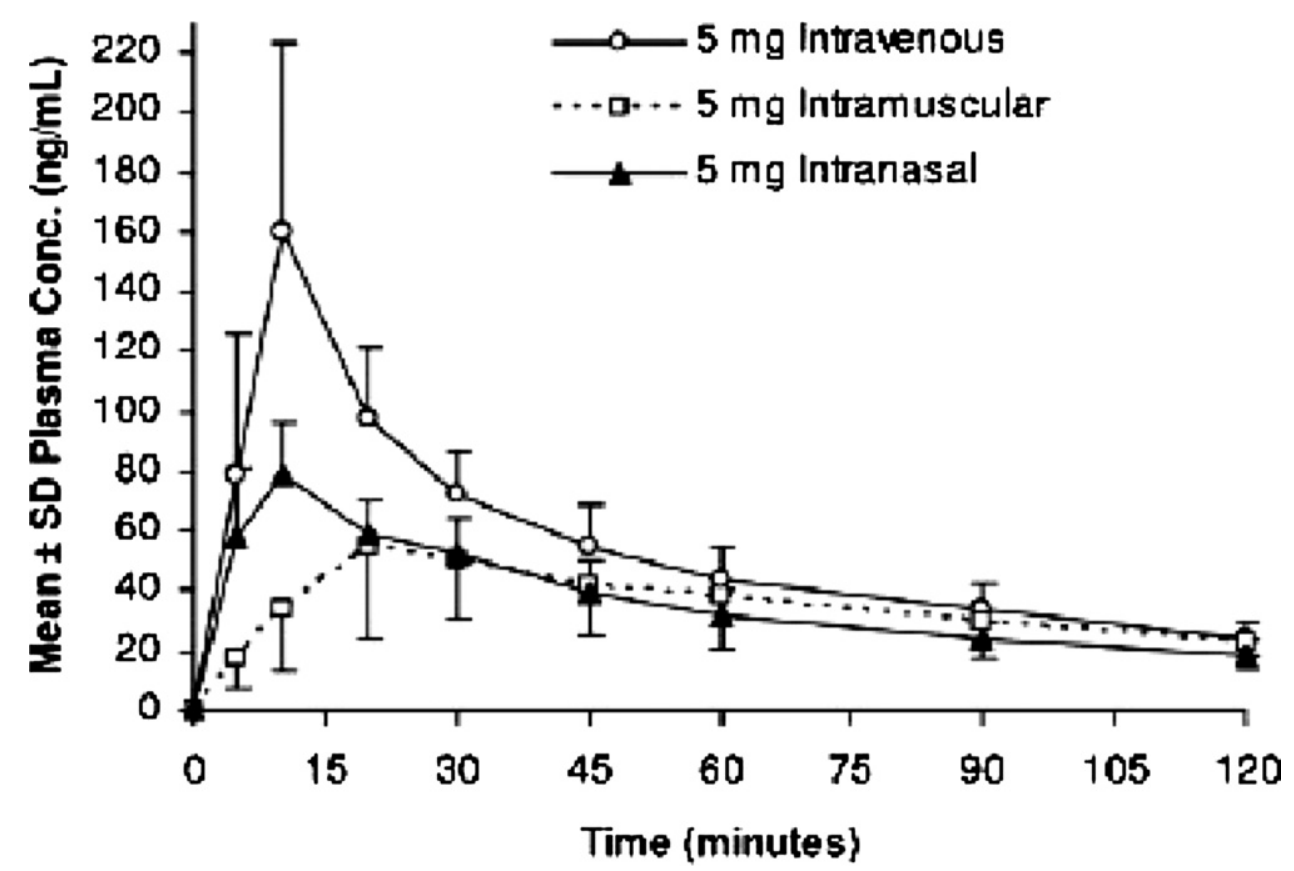

FIG. 3. Midazolam plasma concentrations after $5 \mathrm{mg}$ via intravenous, intramuscular, and intranasal administration. SD $=\mathrm{standard}$ deviation. (Adapted from Wermeling, ${ }^{30}$ 2006.)

examines formulation approaches and the resulting pharmacokinetic measurements. ${ }^{25-34}$ The main considerations from these data might be whether maximum plasma concentrations represented by $\mathrm{C}_{\max }$ are within ranges known to produce pharmacologic effects, and the second consideration is whether the concentrations are achieved rapidly enough to be appropriate for treating a seizure. Well-designed nasal preparations may demonstrate a pharmacokinetic profile in which the rate and extent of drug exposure is superior to intramuscular injection and approximates IV infusion (FIG. 3) ${ }^{30}$ The bioavailability, representing the extent of absorption compared to an IV dose may not be as relevant.

The data for intranasal administration of benzodiazepines suggests that certain formulations could be appropriate for treating seizures. The evidence is indirect in the sense that some formulations produce rapid plasma levels that are associated with IV or other routes of administration. Moreover, some studies collected pharmacodynamic information such as psychomotor or cognitive impairment studies, or sedation scoring, in conjunction with pharmacokinetic sampling. The pharmacodynamic data can be compared with dose and concentration data of the original studies of benzodiazepine effects on EEG beta wave $(13-30 \mathrm{~Hz})$ activity. Greenblatt et al. ${ }^{35}$ established that the degree of impairment on digital-symbol substitution tests was directly correlated with the degree of change in new-onset beta wave activity after triazolam administration. Moreover, Lindhardt et al. ${ }^{32}$ demonstrated comparable changes in EEG amplitude in the 16 to $35 \mathrm{~Hz}$ range comparing $4 \mathrm{mg}$ intranasal to $5 \mathrm{mg}$ intravenous di- azepam. Thus, these early studies examining formulation strategy, biopharmaceutics, and local tolerance were suggestive that nasal delivery of benzodiazepines could have a role in treatment of seizures.

\section{EXPERIENCE WITH INTRANASAL DELIVERY OF BENZODIAZEPINES FOR TREATMENT OF SEIZURES}

Studies demonstrating nasal delivery of midazolam to treat seizures, as compared with other benzodiazepines, are by far the most prevalent in the biomedical literature. O'Regan et al. ${ }^{36}$ was the first to publish success using this method by administering $0.2 \mathrm{mg} / \mathrm{kg}$ of the midazolam injection solution intranasally. The results demonstrated successful abolition of seizures in 14 of 19 children with difficult to treat seizures. There was a $60 \%$ reduction in spike counts per minute and the appearance of beta-wave activity within 175 seconds of drug administration.

Many additional articles describe intranasal delivery of midazolam in case reports or open-label studies with relatively small numbers of patients. However, in aggregate, the studies are universally positive in their patient outcomes of rapid treatment and cessation of seizures. Three articles of similar construct compared intranasal midazolam with intravenous diazepam for cessation of seizures rates and time to cessation. ${ }^{37-39}$ Each research team reached the same conclusions: 1) midazolam injection administered nasally was equally effective as IV diazepam in seizure cessation, and 2) intranasal midazo- 
lam reduced time to cessation of seizures by eliminating the need to establish IV access.

Bhattacharyya et al. ${ }^{40}$ conducted a controlled trial of intranasal midazolam to rectal diazepam in children. There was a shorter time to seizure cessation in the midazolam group. Intranasal midazolam had a superior tolerability profile with lower incidences of drowsiness, nausea, and respiratory depression.

Similar outcomes have been reported when 0.2 $\mathrm{mg} / \mathrm{kg}$ IV midazolam administered intranasally was compared with rectal diazepam in children in the prehospital setting. ${ }^{21}$ In addition to the aforementioned advantages from Bhattacharyya et al., ${ }^{40}$ the investigators demonstrated that midalozam-treated patients were less likely to be seizing on presentation to the emergency department, needed less additional seizure medication, had a lower incidence of intubation, or were less likely to be admitted to the hospital or intensive care unit.

Intranasal drug delivery has been readily accepted by the layman as demonstrated by the considerable usage of over-the-counter and prescription products. Nasal drug delivery of midazolam for treatment of seizures in the home-bound setting has been successful in preliminary studies. ${ }^{41-43}$ Clearly, morbidity and mortality, the intensity of health-care resources and expenses associated with treatment of epileptic patients could be reduced if an effective and safe transmucosal treatment was available for use by caregivers.

Lorazepam injection administered by intranasal spray was compared to intramuscular paraldehyde for protracted seizures in children of sub-Saharan Africa. ${ }^{44}$ Children randomized to lorazepam received $100 \mathrm{mcg} / \mathrm{kg}$ (drawn up into a syringe with a nasal spray adapter). Seventy-five percent of the lorazepam patients had seizures stop within 10 minutes. The most significant finding may have been that there was a great difference in favor of intranasal lorazepam for the reduction of needing two or more additional rescue anticonvulsant agents. The authors believe their intranasal lorazepam system is an ideal primary health-care facility, first-line anticonvulsant agent, by satisfying the following criteria: quick acting, minimal cardiopulmonary side-effects, long-lasting effect, and inexpensive.

Intranasal delivery of benzodiazepines has been recommended in treatment guidelines when IV access has not yet been established. Table 3 provides a general guideline that clinicians may wish to consider if nasal delivery of benzodiazepines is to be used in their practice. Many of the key concepts are directly provided from the literature. Most importantly, development of protocols for nonphysicians will assist in keeping this a safe drug-delivery practice.
Table 3. Key Concepts for Clinical Use of Intranasal Benzodiazepine Delivery

- The choice of medication based on pharmacology, regulatory, and clinical experience

- Highly concentrated, stable drug solution

- 100-200 uL delivery volume per spray for commercial products

- As concentrated as available for injection solution products

- Choose an appropriate nasal spray device

- Atomizers distribute spray across the nasal mucosa

- Use both naris to increase absorption surface area

- Desirable pharmacokinetic and pharmacodynamic profiles

- Rapid absorption

- Sufficient duration to permit dosing of other medications

- Develop assessment, administration, and monitoring protocols and training guides

- Emergency room and transport settings

- Home-bound setting for patients and caregivers

\section{CONCLUSIONS}

Benzodiazepines, although generally water-insoluble, can be designed into appropriate nasal delivery candidates. Preparations for clonazepam, diazepam, lorazepam, and midazolam have been described. Clinical researchers have investigated with considerable success the potential for injectable benzodiazepine solutions to serve as a surrogate for optimally designed nasal spray formulations to treat seizures. The availability of lowtech nasal spray devices has facilitated this research and permitted translation of this technique into emergency medicine practice in some locales. Midazolam, perhaps due to its rapid absorption and action in the CNS, appears to be the medication of choice at this time. Lorazepam has been shown to be a suitable alternative if recurrence of seizures is a potential concern. Additional research is needed to better define the optimal dose of these medications for nasal administration. Although promising results have been achieved, additional research into the utility of this technique for home-bound treatment is also warranted.

The emergency medical community is striving to improve the care of the seizure patient by examining the drug-delivery techniques to reduce the time to treatment and cessation of seizures. The literature demonstrates an unmet medical need exists to provide well-designed nasally delivered benzodiazepines for the treatment seizures.

\section{REFERENCES}

1. Lowenstein DH, Alldredge BK. Status epilepticus. N Eng J Med 1998;338:970-976.

2. Lowenstein DH, Bleck T, Macdonald RL. It's time to revise the definition of status epilepticus. Epilepsia 1999;40:120-122.

3. Wang HC, Chang WN, Chang HW, et al. Factors predictive of outcome in posttraumatic seizures. J Trauma 2008;64:883-888. 
4. Stavem K, BJornaes H, Langmoen IA. Long-term seizures and quality of life after epilepsy surgery compared with matched controls. Neurosurgery 2008;62:326-334.

5. Logroscino G, Hessdorffer DC, Cascino G, et al. Time trends in incidence, mortality, and case-fatality after first episode of status epilepticus. Epilepsia 2001;42:1031-1035.

6. Feen ES, Bershad EM, Suarez JI. Status Epilepticus. South Med J 2008;101:400-406.

7. Pang T, Hirsch L. Treatment of convulsive and nonconvulsive status epilepticus. Cur Treat Options Neurol 2005;7:247-259.

8. Smith B. Treatment of status epilepticus. Neurol Clin 2001;19: 347-369.

9. Ericksson K, Kalviainen R. Pharmacologic management of convulsive status epilepticus in childhood. Expert Rev Neurotherapeutics 2005;5:777-783.

10. Wolfe T, Macfarlane T. Intranasal midazolam therapy for pediatric status epilepticus. Am J Emerg Med 2006;24:343-346.

11. Meierkord H, Engelsen B, Gocke K, et al. EFNS guideline on the management of status epilepticus. Eur J Neurol 2006;13:445-450.

12. Prasad K, Krishman P, Al-Roomi K, et al. Anticonvulsant therapy for status epilepticus. Br J Clin Pharmacol 2007;63:640-647.

13. Illum L. Nasal Clearance in Health and Disease. J Aerosol Med 2006;19:92-99.

14. Merkus P, Ebbens FA, Muller B, Fokkens WJ. Influence of anatomy and head position on intranasal drug deposition. Eur Arch Otorhinolaryngol 2006;263:827-832.

15. Illum L. Transport of drugs from the nasal cavity to the central nervous system. Eur J Pharm Sci 2000;11:1-18.

16. Illum L. Is nose to brain transport of drugs a reality? JPP 2004; $56: 3-17$.

17. Merkus F, van den Berg MP. Can nasal drug delivery bypass the blood-brain barrier? Questioning the direct transport theory. Drugs 2007;8:133-144.

18. Constantino HR, Illum L, Brandt G, et al. Intl J Pharmaceutics 2007;337:1-24

19. Davis GA, Rudy AC, Archer SA, Wermeling DP, McNamara PJ. Effect of fluticasone propionate nasal spray on bioavailability of hydromorphone hydrochloride in patients with allergic rhinitis. Pharmacotherapy 2004;24:26-32.

20. Davis GA, Rudy AC, Archer SM, Wermeling DP, McNamara PJ. Bioavailability and pharmacokinetics of intranasal hydromorphone in patients experiencing vasomotor rhinitis. Clin Drug Invest 2004; 24:1-7.

21. Holsti M, Sill B, Firth S, et al. Prehospital intranasal midazolam for the treatment of pediatric seizures. Ped Emerg Care 2007;23:148153.

22. Rudy AC, Coda BA, Archer SM, Wermeling DP. A multiple dose phase 1 study of intranasal hydromorphone hydrochloride in healthy volunteers. Anesth Analg 2004;99:1379-1386.

23. Van den Donk HJ, van den Huervel AG, Zuidema J, Merkus FW. The effects of nasal drops and their additives on human nasal mucociliary clearance. Rhinology 1982;20:127-137.

24. Kubek MJ, Domb AJ, Veronesi MC. Attenuation of kindled seizures by intranasal delivery of neuropeptide-loaded nanoparticles. Neurotherapeutics 2009;6:359-371.

25. Loftsson T, Gudmundsdottir H, Sigurjonsdottir JF, et al. Cyclodextrin solubilization of benzodiazepines: formulation of midazolam nasal spray. Int J Pharm 2001;212:29-40.

26. Knoester PD, Jonker DM, Van Der Hoeven RT, et al. Pharmacokinetics and pharmacodynamics of midazolam administered as a concentrated intranasal spray. A study in healthy volunteers. Br J Clin Pharmacol 2002;53:501-507.

27. Gudmundsdottir H, Sigurjonsdottir JF, Masson M, et al. Intranasal administration of midazolam in a cyclodextrin based formulation: bioavailability and clinical evaluation in humans. Pharmazie 2001; 56:963-966.

28. Dale O, Nilsen T, Loftsson T. Intranasal midazolam: a comparison of two delivery devices in human volunteers. J Pharmacy Pharmacol 2006;58:1311-1318.

29. Burstein AH, Modica R, Hatton M, et al. Pharmacokinetics and pharmacodynamics of midazolam after intranasal administration. J Clin Pharmacol 1997;37:711-718.

30. Wermeling D, Record K, Kelly T, et al. Pharmacokinetics and pharamcodynamics of a new intranasal midazolam formulation in healthy volunteers. Anesth Analg 2006;103:344-349.

31. Schols-Hendriks MWG, Lohman JJHM, Janknegt R, et al. Absorption of clonazepam after intranasal and buccal administration. Br J Clin Pharmacol 1995;39:449-451.

32. Lindhardt K, Gizurarson S, Stefansson SB, et al. Electroencephalographic effects and serum concentrations after intranasal and intravenous administration of diazepam to healthy volunteers. Br J Clin Pharmacol 2001;52:521-527.

33. Lau SWJ, Slattery JT. Intl J Pharmaceutics. Absorption of diazepam and lorazepam following intranasal administration. Int J Pharmaceutics 1989;54:171-174.

34. Wermeling DP, Miller JL, Archer SM, et al. Bioavailability and pharmacokinetics of lorazepam after intranasal, intravenous, and intramuscular administration. J Clin Pharmacol 2001;41: 1225-1231.

35. Greenblatt D, Gan L, Harmatz, et al. Pharmacokinetics and pharmacodynamics of single-dose triazolam: EEG compared with digital symbol substitution test. Br J Clin Pharmacol 2005;60:244248.

36. O'Regan M, Brown J, Clarke M. Nasal rather than rectal benzodiazepines in the management of acute childhood seizures. Develop Med and Child Neurol 1996;38:1037-1045.

37. Mittal P, Manohar R, Rawat A. Comparative study of intranasal midazolam and intravenous diazepam sedation for procedures and seizures. Ind J Pediatrics 2006;73:975-978.

38. Mahmoudian T, Zadeh M. Comparison of intranasal midazolam with intravenous diazepam for treating acute seizures in children. Epilepsy Behav 2004;5:253-255.

39. Lahat E, Goldman M, Barr J, et al. Comparison of intranasal midazolam with intravenous diazepam in treating febrile seizures in children: prospective randomized study. BMJ 2000;321:83-87.

40. Bhattacharyya M, Kalra V, Gulati S. Intranasal midazolam vs rectal diazepam in acute childhood seizures. Pediatric Neurology 2006;34:355-359.

41. Wilson MT, Macleod S, O'Regan ME. Nasal/buccal midazolam use in the community. Arch Dis Child 2004;89:50-51.

42. Harbord MG, Kyrkou NE, Kyrkou MR, Kay D, Coulthard KP. Use of intranasal midazolam to treat acute seizures in paediatric community settings. J Paediatr Child Health 2004;40:556-558.

43. Jeannet PY, Roulet E, Maeder-Ingvar M, Gehri M, Jutzi A, Deonna T. Home and hospital treatment of acute seizures in children with nasal midazolam. Eur J Paediatr Neurol 1999;3:73-77.

44. Ahmad S, Ellis J, Kamwendo H, Molyneux E. Efficacy and safety of intranasal lorazepam versus intramuscular paraldehyde for protracted convulsions in children: an open label trial. Lancet 2006; 367:1591-1597. 